\title{
An Improved EZW Hyperspectral Image Compression
}

\author{
Kai-Jen Cheng ${ }^{*}$, Jeffrey C. Dill* \\ School of Electrical Engineering and Computer Science, Ohio University, Athens, USA. \\ Email: ${ }^{* k} 134905 @ o h i o . e d u ;$ dill@ohio.edu
}

Received October 2013

\begin{abstract}
The paper describes an efficient lossy and lossless three dimensional (3D) image compression of hyperspectral images. The method adopts the 3D spatial-spectral hybrid transform and the proposed transform-based coder. The hybrid transforms are that Karhunen-Loève Transform (KLT) which decorrelates spectral data of a hyperspectral image, and the integer Discrete Wavelet Transform (DWT) which is applied to the spatial data and produces decorrelated wavelet coefficients. Our simpler transform-based coder is inspired by Shapiro's EZW algorithm, but encodes residual values and only implements dominant pass incorporating six symbols. The proposed method will be examined on AVIRIS images and evaluated using compression ratio for both lossless and lossy compression, and signal to noise ratio (SNR) for lossy compression. Experimental results show that the proposed image compression not only is more efficient but also has better compression ratio.
\end{abstract}

\section{KEYWORDS}

Wavelet Transform; Karhunen-Loève Transform; Transform-based Image Compression; AVIRIS Hyperspectral Image; Embedded Zerotree Wavelet

\section{Introduction}

Hyperspectral images are widelyused in various fields such as agriculture, topography, meteorology and military, since they can provide more accurate and detailed spectral information than other images. NASA Jet Propulsion Laboratory developed Airborne Visible/Infrared Imaging Spectrometer (AVIRIS) to produce the 614 pixel $\times 512$ line hyperspectral images in 224 contiguous spectral bands with wavelengths from 400 to 2500 nanometers (nm) [1]. The spectrometer generates huge amounts of data and causes that distribution facilities cannot economically handle this level of data. It is imperative to perform compression.

One popular and widely used image compression is transform-based compression which successful incorporates the useful statistical properties of transform for image compression such as energy compaction and decorrelated components. Examples of transforms include the Karhunen-Loève Transform (KLT), Discrete Fourier Transform (DFT), Discrete Cosine Transform (DCT) and Discrete Wavelet Transform (DWT). In general, the standard image compression, Joint Photographic Experts Group (JPEG), uses 8 × 8 DCT and the later JPEG2000 uses 2D DWT. Penna et al. [2] compressed hyperspectral

${ }^{*}$ Corresponding author. images using JPEG2000 and investigated the performance under different transform techniques including WT, DCT, KLT, and various combinations.

Shapiro [3] proposed the classic embedded zerotree coding (EZW) using wavelet transform to compress images. Bilgin et al proposed three-dimensional (3D) image compression algorithm [4].The 3D-SPIHT was proposed by Kim and Pearlman [5]. Sohn and Lee [6] successfully applied the 3D-SPIHT algorithm with symmetrical 3DDWT to hyperspectral images.

The proposed image compression utilizes the 3D space-spectral: The Karhunen-Loève Transform (KLT) is first applied to remove the correlations among the 224 contiguous highly correlated spectral bands. 2D-Discrete Wavelet Transform (DWT) is applied to remove the spatial redundancies. After the hybrid transform, the modified EZW algorithm is applied to encode the transformed hyper spectral images. The modifications include defining a new tree structure, only running the dominant pass using six symbols and coding residual values. Figure 1 shows a block diagram of the transform-base- dimage compression. The remainder of the paper is organized as follows: Section 2 describes the integer KLT. Section 3 discusses the 2D integer DWT. In Section 4, the modified EZW algorithm for hyperspectral images is introduced. The last three sections describe the testing of 


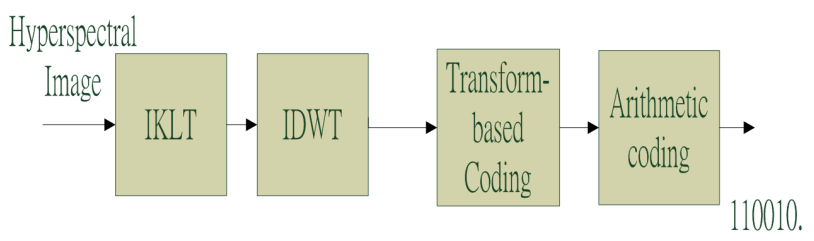

Figure 1. System diagram.

the algorithms using AVIRIS hyperspectral images; provide results for this proposed hyperspectral image compression and conclusions.

\section{Integer Karhunen-Loève Transform (KLT)}

The Karhunen-Loève Transform (KLT) is the data dependent and optimal linear orthogonal transform because of the eigenvectors matrix derived from the covariance matrix of the data.

The pixel vector at the $i^{\text {th }}$ band is expressed as $A_{i}=\left[a_{i 1}, a_{i 2}, \ldots \ldots \ldots \ldots, a_{i M}\right]$ in the spatial dimension (x-y plane). If the spatial dimension has $m$ rows and $n$ columns, the length of the vector is equal to $M=m \times n$. The number of $A_{i}$ is same as the hyperspectral bands $N$, namely $i=1 \ldots N$. Figure 2 demonstrates the perspective of the pixel vector in the hyperspectral image.

The covariance matrix is derived from the pixel vectors as follows:

$$
C_{a}=E\left\{(A-\bar{a})(A-\bar{a})^{T}\right\}
$$

where $\mathrm{E}\{\cdot\}$ is the expected value of the argument, $T$ is matrix transpose and the $\overline{\boldsymbol{a}}$ is defined as the mean value of $A$, as in

$$
\bar{a}=\sum_{i=1}^{M} a_{i} / M
$$

Since the covariance matrix is a real and symmetric $N \times N$ square matrix, the eigenvectors and eigenvalues for this matrix can be found [7].

In general, once eigenvectors are found from the covariance matrix, the next step is to order them by eigenvalues, highest to lowest. This gives you the components in order of signficance. If applying the ordered eigenve ctors to the image, two important features are presented in the spectral dimensions and they are the decorrelated spectral data, and the energy compaction. Figure 3 depicts the energy compaction in the spectral dimension. The optimal energy distribution declines monotonically from the first band to the bottom. After KLT, we call these bands KLT bands. IKLT bands mean that integer KLT (IKLT) is implemented by mapping integers to integers as described below.

In order to attain lossless compression, the reversible integer KLT is utilized because it is able to map integers to integers. Based on matrix factorizations [8], the nonsingular eigenvectors of the KLT is factorized as $\mathbf{A}=$

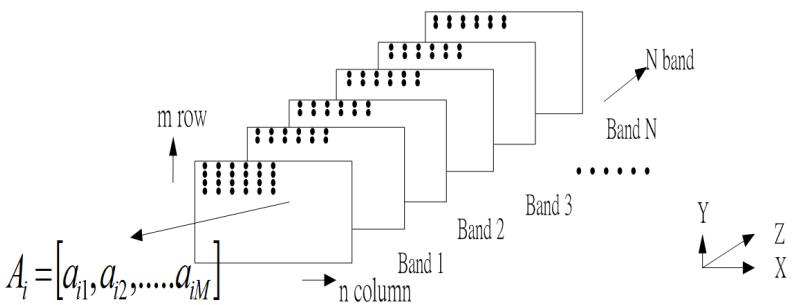

Figure 2. The perspective of the pixel vector in the hyperspectral image.

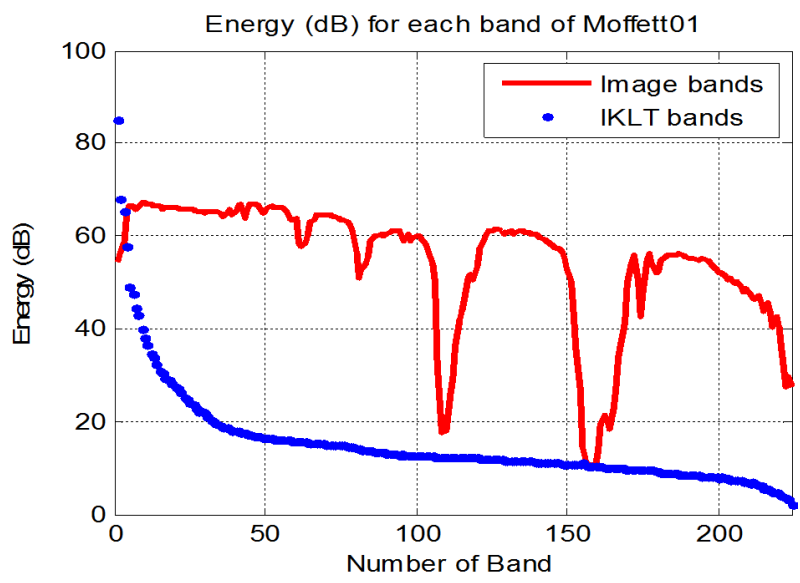

Figure 3. Energy compaction in spectral dimensions.

PLUS where $\boldsymbol{L}$ and $\boldsymbol{S}$ are lower Triangular Elementary Reversible Matrices (TERM), $\boldsymbol{U}$ is upper TERM, and $\boldsymbol{P}$ is a reversible permutation matrix. $\boldsymbol{P}$ defines the row interchanges to guarantee diagonal elements that are not zero. The transformation can be implemented by the lifting scheme, which includes operations of multiplication, addition and rounding up.

These factorized matrices must be recorded as overhead information for the reverse transform. Even if not compressed, representing these matrices as 32-bit floating point numbers computes an overhead of about 0.11 bpppbs for the $224 \times 224$ matrices. In the experiment section, we include the overhead into the lossless results in each table. Figure 3 describes that the integer KLT is optimal transform in terms of optimal energy compaction and best decorrelation.

\section{2D-Wavelet Transform}

Instead of implementing the 3D discrete wavelet transform (DWT), the following step is to transform the image in the spatial dimension by the use of 2D DWT. The optimal implementation of the 2D-DWT is the lifting scheme. It not only has less computational complexity, but also realizes a reversible integer wavelet transform [9].

In our experiments, the 3D hyperspectral image was decomposed by the 2D dyadic wavelet Band by Band, separately. Figure 4 depicts the perspective of the second 


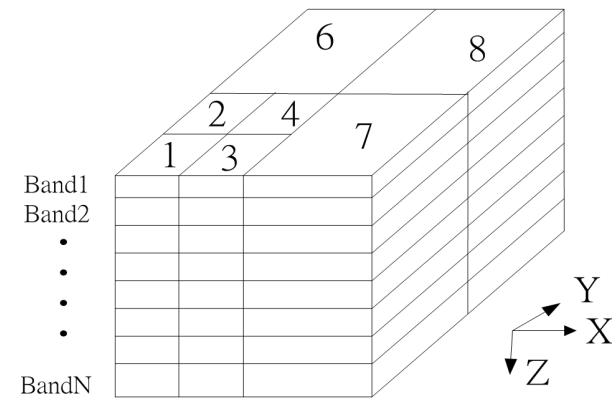

Figure 4. 2-D wavelet transform coefficients are stored in a data cube.

level decomposition on each band.

The DWT has two important properties which are critical for image compression:

1) Energy packing: When an image is wavelet transformed, the transformed image has energy compaction in spectral dimensions, that is, the wavelet coefficients in the higher level subbands will, on average, be larger than those in the lower level subbands.

2) Self-similarity: A wavelet coefficient at a higher level subband and all wavelet coefficients of the same spatial orientation at lower level subbands have certain predictable relationships.

\section{Shapiro's EZW Algorithm}

Shapiro invented his Embedded Zerostree Wavelet (EZW) algorithm taking advantage of the wavelet transform [2]. The EZW algorithm implements a progressive, embedded image coding method based on the zerotrees of data structure. All currently significant bits at the same bitplane together and recursively encodes other pixels for the next significant bitplane until reaching the least significant bitplane. As a result, the lower significant bits are embedded behind the higher significant bits, so that a decoder quickly displays a low quality image and better quality as more bits are received.

We notethree crucial components that make Shapiro's EZW algorithm effectivein image compression. First, due to energy packing, these wavelet coefficients in higher level subbands could be scanned earlier than others in low level subbands. In other words, larger coefficients will be encoded first. In addition, both receiver and transmitter know what scanning order is selected such that it does not include the scanning order in the overhead. Scanning order used in this paper is Morton scan.

Second, the quad-tree is the fundamental idea of the EZW algorithm to interpolate the relations among wavelet coefficients in different subbands; therefore, it is set up based on the self-similarity. The definition of the quad-tree was introduced in [2].

There are two steps to complete EZW algorithm: thedominant pass and the subordinate pass. The dominant pass keeps track of the search for significant coefficients by labeling each pixel among these four labels: significance positive symbol (POS), significant negative symbol (NEG), zerotree root (ZTR) and isolated zeros (IZ) in Figures 5(a)-(c). The subordinate pass quantizes each significant coefficient that has been found in the dominant pass.

The definitions of four symbols are described below. If a root coefficient, in absolute value, is larger than a threshold, it is labeled as significant positive (POS) or significant negative (NEG) in Figure 5(b). It implies that some of the coefficients' descendants are significant. An isolated zero (IZ) is a root coefficient that is insignificant but has some significant descendants in Figure 5(c). If the coefficient is zerotree root (ZTR), it means the root coefficient itself and its descendants are all insignificant such that these descendants don't have to be encoded in the current iteration in Figure 5(a).

\section{Modified EZW Algorithm}

We propose some modifications to simplify the convention EZW algorithm and improved the compression results. [10-13] have studied the asymmetrical 3D-DWT decomposition that causes the asymmetrical statistics of the transformed hyperspectral image; thus the asymmetrical tree structure is more suitable for describing the transformed hyperspectral image. In this paper, the 3D asymmetric tree structure was designed according to the properties of hybrid transforms. In Figure 4, a wavelet coefficient at a higher subband is not only relative to all wavelet coefficients of the same spatial orientation at lower subbands at the same band but also in the neighbor band. Therefore, if the approximation subband is $\left[m / 2^{l}\right]$-by- $\left[m / 2^{l}\right]$ with $l$-level decomposition at the first band, while the spatial dimensions of the image are m-by-n. Any root $(x, y, 0)$ in the approximation has four immediate children at $\left(x+m / 2^{l}, y, 0\right)$,

$\left(x, y+m / 2^{l}, 0\right),\left(x+m / 2^{l}, y+m / 2^{l}, 0\right)$ and $(x, y, 1)$ In addition, except ones in the approximation, any root (x, $y, 0)$ at the first band has four children located at the same spatial orientations $(2 x, 2 y, 0),(2 x+1,2 y, 0),(2 x$, $2 y+1,0)$, and $(2 x+1,2 y+1,0)$ on the first band and one more child $(\mathrm{x}, \mathrm{y}, 1)$ below it. Note that any pixel not in the first band has only one child below it. The new tree structure will continue to branch until no offspring can be found. The new and simple definition of tree structure is demonstrated in Figure 6. Each pixel in the low-pass (approximation) section of band 1 is a tree root.

In addition to the four labels defined in Shapiro's EZW algorithm, in this study, we define two more labels, called positive and negative ZTR (PZT and NZT) in Figure 5(d). According to [14], they are degree-1 zerotrees since every coefficient except the root coefficient is all zeros. Roots of PZT and NZT are positive and nega- 


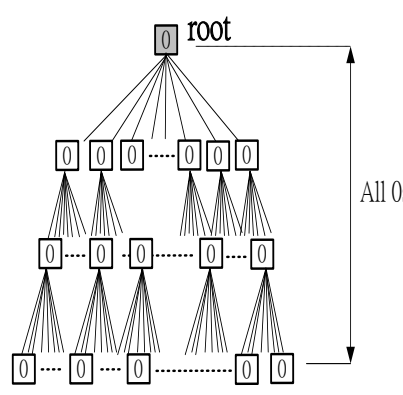

(a)

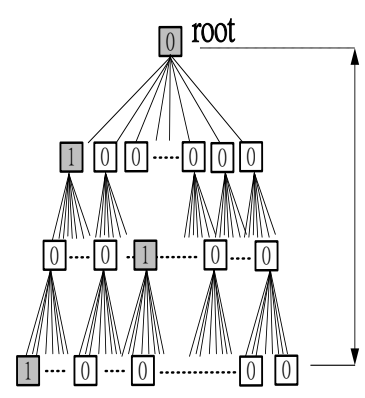

(c)

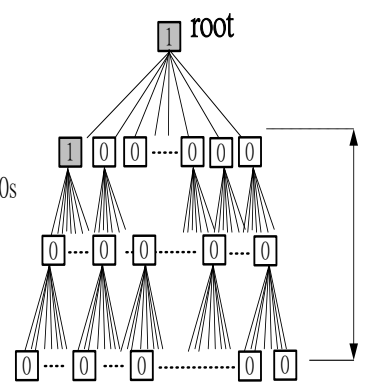

(b)

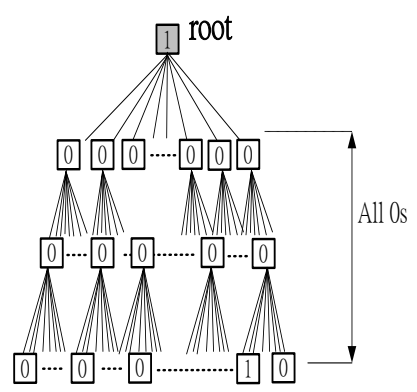

(d)
Figure 5. Explanation of (a) ZRT (b) POS/NEG (c) IZ and (d) PZT/NZT.

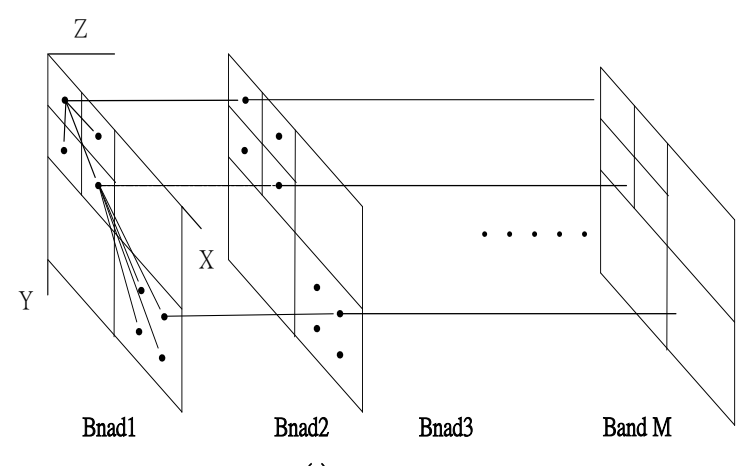

(a)

Figure 6. A new tree structure: The root has three children at the same band and one child at the lower band.

tive significant, respectively. A higher degree zero tree coder generates a shorter symbol stream.

Table 1 shows the coding results of tree (b) and (d) in Figure 5. Any significant coefficient should do further search on its child nodes such that there are eight more symbols generated for each POS/NEG symbol. If applied to the new PZT/NZT symbols, some redundant symbols for coding tree (d) can be replaced by one PZT symbol, which saves an extra eight symbols. This is the main advantage of adding extra symbols (PZT/NZT).

Table 2 demonstrates that the conventional EZW algorithm encodes the image, Jasper, with and without PZR/NZT. Their performances are studied in terms of the total numbers of outputs. Basically, the PZR/NZR is
Table 1. Examples of coded symbols generated by EZW algorithm for tree (b) and (d).

\begin{tabular}{cc}
\hline & EZW \\
\hline Coding & POS, \\
tree (b) & POS, ZRT, ZRT, ZRT, ZRT, ZRT, ZRT, ZRT, ZRT, \\
& ZRT, ZRT, ZRT, ZRT, ZRT, ZRT, ZRT. \\
Coding & POS, \\
tree (d) & ZRT, ZRT, ZRT, ZRT, ZRT, ZRT, ZRT, ZRT \\
Coding tree (d) & PZT \\
\hline
\end{tabular}

Table 2. Numbers of coded symbols from the conventional EZW algorithm with PZR/NZT and without PZR/NZT, using the bior-4.4 filter on Jasper.

\begin{tabular}{ccc}
\hline $\begin{array}{c}\text { Number of } \\
\text { symbols }\end{array}$ & $\begin{array}{c}\text { EZW with } \\
\text { PZR/NZT }\end{array}$ & $\begin{array}{c}\text { EZW without } \\
\text { PZR/NZT }\end{array}$ \\
\hline Num of ZRT & $9,265,805$ & $10,584,445$ \\
Num of PZR & 1633198 & NA \\
Num of NZR & 1585476 & NA \\
Num of POS/NEG & 278442 & 3497116 \\
Num of IZ & 1505055 & 1505055 \\
\hline
\end{tabular}

parts of the POS/NEG symbols. The total number of PZR/NZR and POS/NEG should be equal to that of the POS/NEG from the EZW algorithm without PZR/NZT, that is, $1633198+1585476+278442=3497116$ in Table 2. Compared with the numbers of ZRT symbol, adding extra symbols help to reduce 1,318,640 ZRT symbols in Table 2. Overall, the total symbols are reduced and the result in better compression ratios.

Moreover, in Shapiro's EZW algorithm, if a coefficient is recognized as a significant pixel, the coefficient will be sent to the subordinate list and set to 0 . Table 3 lists the bits rates generated from the dominant and subordinate passes and shows that the subordinate pass contributes on average one-third of the total bit rate. In order to improve the compression ratio, we consider removing the subordinate list such that the significant coefficient is replaced by a residual value.

$$
R(x, y, z)=|I(x, y, z)|-T_{i}
$$

where:

$R(x, y, z)$ is residual value at $(\mathrm{x}, \mathrm{y}, \mathrm{z})$

$T_{i}$ is threshold at the $i^{\text {th }}$ iteration

$|I(x, y, z)|$ is absolute value of a pixel at $(\mathrm{x}, \mathrm{y}, \mathrm{z})$

This is a simple way to replace the subordinate list, but still complete the job of quantizing coefficients. In sum, the outputs of the modified EZW algorithm only have the symbols stream that contains a sequence of symbols (POS, NEG, ZTR, PZT, NZT and IZ). At this point, the proposed method we call is the residual EZW algorithm incorporating with the PZT/NZT symbols and the new tree structure. 
Table 3. Comparison of the bit rates generated by the conventional EZW algorithm using new $3 D$ asymmetric trees and the hybrid transform.

\begin{tabular}{cccc}
\hline & \multicolumn{3}{c}{ Compression performance (bpppbs) } \\
\cline { 2 - 4 } & Jasper & Moffett & Low Altitude \\
\hline Dominant pass & 4.23 & 4.16 & 4.26 \\
Subordinate pass & 1.64 & 1.48 & 1.62 \\
Total bit rate & 5.87 & 5.64 & 5.88 \\
\hline
\end{tabular}

\section{Experimental Results}

The residual EZW algorithm compresses the first scene of AVIRIS images, Moffett 01, Jasper 01 and Low Altitude 01 . The size of each image is $256 \times 256 \times 224$ and is stored as 16 bits signed integers. Compression ratio is the bit per pixel per band (bpppb). The rate-distortion performance is expressed as the signal to noise ratio (SNR) for various bit rates. The definition of SNR is the average squared value of the original AVIRIS images is divided by the mean squared error (MSE).

First, Table 4 demonstrates the lossless performance of the residual EZW algorithm along with various wavelet filters. They are Daubechies wavelets $(\mathrm{db} N)$, Symlets $(\operatorname{sym} N)$ ), and Biorthogonal wavelets (biorNr. Nd), which can be found in the Matlab wavelet toolbox. As a result, there is no the optimal wavelet filter for all test images. General speaking, the biorthogonal wavelets are more suitable for compressing images because of the perfect symmetry and linear phase.

Second, the lossy performance of the selected conventional technique (3D-SPIHT and 3D-EZW) is compared to the proposed method. Both methods are based on the symmetrical 3D-DWT and 3D quad-tree. For lossy compression, the transform-based coder can be stopped at a predetermined threshold or when the bit budget is reached. Table 5 to Table 7 show all results (bpppbvs SNR) of the lossless, and lossy compression based on predetermined thresholds. All images are decomposed by 4-level bior 4.4 filters, which have better performance for lossy compression. The results show that the proposed EZW algorithm outperforms two conventional methods. Tables 5 and $\mathbf{7}$ shows that the proposed method consistently has lower compression ratios and relative high SNRs.

We demonstrate the complexity in terms of simulation times (s) on a workstation with Intel(R) Core i5 CPU $2.67 \mathrm{GHz}$ processor and Windows 7 operating system. All algorithms, compressing the hyperspectral images, are implemented in MATLAB. Table 8 shows that our proposed EZW using 6 symbols has a moderate computational complexity and achieves an improved compression ratio. Therefore, the residual EZW algorithm is an efficient lossy and lossless compression algorithm for hyperspectral images.
Table 4. Lossless compression results (bpppbs) of the residual EZW algorithm with PZT/NZT using new 3D asymmetric trees and hybrid transform.

\begin{tabular}{cccc}
\hline \multirow{2}{*}{ Wavelet } & \multicolumn{3}{c}{ Compression performance (bpppb) } \\
\cline { 2 - 4 } & Jasper & Moffett & Low Altitude \\
\hline Db 2 & 5.57 & 5.42 & 5.72 \\
Db 6 & 6.59 & 6.48 & 6.66 \\
Sym 4 & 5.49 & 5.34 & 5.63 \\
Sym 6 & 6.53 & 6.44 & 6.56 \\
Bior 3.5 & 5.58 & 5.41 & 5.74 \\
Bior 1.3 & 5.38 & 5.22 & 5.53 \\
Bior 1.5 & 5.40 & 5.24 & 5.55 \\
Bior 4.4 & 5.47 & 5.29 & 5.56 \\
Bior 2.4 & 5.34 & 5.17 & 5.49 \\
Bior 2.6 & $\mathbf{5 . 3 3}$ & $\mathbf{5 . 1 6}$ & $\mathbf{5 . 4 8}$ \\
\hline
\end{tabular}

Table 5. Rate distortion (bpppb vs SNR) of various techniques for Moffett 01.

\begin{tabular}{cccccc}
\hline \multirow{2}{*}{$\begin{array}{c}\text { Bitplane } \\
\text { Cutoff Algorithm }\end{array}$} & $\mathbf{0}$ & $\mathbf{1}$ & $\mathbf{2}$ & $\mathbf{4}$ & $\mathbf{8}$ \\
\cline { 2 - 6 } & 7.91 & 6.51 & 5.01 & 3.579 & 2.41 \\
3D-EZW & 6.38 & 5.30 & 4.15 & 2.97 & 1.95 \\
3D-SPIHT & NA & 51.06 & 48.25 & 44.09 & 39.10 \\
SNR(dB) & $\mathbf{5 . 4 7}$ & $\mathbf{3 . 9 4}$ & $\mathbf{2 . 5 3}$ & $\mathbf{1 . 3 6}$ & $\mathbf{0 . 5 5}$ \\
Residual -EZW & NA & 55.00 & 52.69 & 49.71 & 47.02 \\
SNR(dB) & & & & &
\end{tabular}

Table 6 Rate distortion (bpppb vs SNR) of various techniques for Jasper 01.

\begin{tabular}{cccccc}
\hline \multirow{2}{*}{$\begin{array}{c}\text { Bitplane } \\
\text { Cutoff Algorithm }\end{array}$} & $\mathbf{0}$ & $\mathbf{1}$ & $\mathbf{2}$ & $\mathbf{4}$ & $\mathbf{8}$ \\
\cline { 2 - 6 } & 8.02 & 6.61 & 5.13 & 3.70 & 2.53 \\
3D-EZW & 6.42 & 5.34 & 4.19 & 3.01 & 2.01 \\
3D-SPIHT & NA & 54.21 & 51.42 & 47.29 & 42.28 \\
SNR(dB) & $\mathbf{5 . 2 9}$ & $\mathbf{4 . 1 1}$ & $\mathbf{2 . 7 1}$ & $\mathbf{1 . 4 7}$ & $\mathbf{0 . 7 0}$ \\
Residual -EZW & $\mathbf{5 A}$ & 57.23 & 54.92 & 51.83 & 48.53 \\
SNR(dB) & NA
\end{tabular}

Table 7. Rate distortion (bpppb vs SNR) of various techniques for Jasper 01.

\begin{tabular}{cccccc}
\hline \multirow{2}{*}{$\begin{array}{c}\text { Bitplane } \\
\text { Cutoff Algorithm }\end{array}$} & \multicolumn{5}{c}{ Low Altitude 01 } \\
\cline { 2 - 6 } & $\mathbf{0}$ & $\mathbf{1}$ & $\mathbf{2}$ & $\mathbf{4}$ & $\mathbf{8}$ \\
\hline 3D-EZW & 8.28 & 6.87 & 5.29 & 3.76 & 2.42 \\
3D-SPIHT & 6.57 & 5.50 & 4.38 & 3.19 & 2.08 \\
SNR(dB) & NA & 54.37 & 51.50 & 47.17 & 42.12 \\
Residual -EZW & 5.56 & 4.26 & 2.86 & 1.53 & 0.66 \\
SNR(dB) & 0 & 54.78 & 52.24 & 48.87 & 45.82 \\
\hline
\end{tabular}


Table 8. Compare the simulation time in second of various techniques for hyperspectral images.

\begin{tabular}{ccc}
\hline Images & Jasper 01 & Moffett 01 \\
Time(s) & 145.49 & 146.29 \\
3D EZW & 116.89 & 120.85 \\
3DSPIHT & $\mathbf{6 6 . 5 8}$ & $\mathbf{6 4 . 6 4}$ \\
Residual -EZW & & \\
\hline
\end{tabular}

\section{Conclusions}

In this paper, we propose a novel transform-based algorithm for lossy and lossless hyperspectral image compression. The introduction of modifications in the residual EZW algorithm results in higher compression ratios and moderate computational complexity. Therefore, it is an efficient image compression for hyperspectral images.

\section{REFERENCES}

[1] AVIRIS-Airborne Visible/Infrared Imaging Spectrometer, 2013. http://aviris.jpl.nasa.gov/.

[2] B. Penna, T. Tillo, E. Magli and G. Olmo, "Transform Coding Techniques for Lossy Hyperspectral Data Compression," IEEE Transactions on Geoscience and Remote Sensing, Vol. 45, 2007, pp. 1408-1421. http://dx.doi.org/10.1109/TGRS.2007.894565

[3] J. M. Shapiro, "Embedded Image Coding Using Zerotrees of Wavelet Coefficients," IEEE Transactions on Signal Processing, Vol. 41, 1993, pp. 3445-3462. http://dx.doi.org/10.1109/78.258085

[4] A. Bilgin, G. Zweig and M. V. Marcellin, "Three-Dimensional Image Compression with Integer Wavelet Transform,” Applied Optics, Vol. 39, 2000, pp. 1799-1814. http://dx.doi.org/10.1364/AO.39.001799

[5] K. Beong-Jo, X. Zixiang and W. A. Pearlman, "Low Bit-Rate Scalable Video Coding with 3-D Set Partitioning in Hierarchical Trees (3-D SPIHT)," IEEE Transactions on Circuits and Systems for Video Technology, Vol. 10, 2000, pp. 1374-1387. http://dx.doi.org/10.1109/76.889025
[6] L. Sunghyun, S. Kwanghoon and L. Chulhee, “Compression for Hyperspectral Images Using Three Dimensional Wavelet Transform," IEEE 2001 International Geoscience and Remote Sensing Symposium, Vol. 1, 2001, pp. 109-111.

[7] A Tutorial on Principal Components Analysis, 2002. http://www.ce.yildiz.edu.tr

[8] H. Pengwei and S. Qingyun, "Matrix Factorizations for Reversible Integer Mapping,” IEEE Transactions on Signal Processing, Vol. 49, 2001, pp. 2314-2324. http://dx.doi.org/10.1109/78.950787

[9] I. Daubechies and W. Sweldens, "Factoring Wavelet Transforms into Lifting Steps,” Journal of Fourier Analysis and Applications, Vol. 4, 1998, pp. 247-269. http://dx.doi.org/10.1007/BF02476026

[10] X. Tang, C. Sungdae and W. A. Pearlman, “3D Set Partitioning Coding Methods in Hyperspectral Image Compression," Proceedings of International Conference on Image Processing, Vol. 3, 2003, pp. 239-242.

[11] E. Christophe, C. Mailhes and P. Duhamel, "Hyperspectral Image Compression: Adapting SPIHT and EZW to Anisotropic 3-D Wavelet Coding," IEEE Transactions on Image Processing, Vol. 17, 2008, pp. 2334-2346. http://dx.doi.org/10.1109/TIP.2008.2005824

[12] G. Liu and F. Zhao, "Efficient Compression Algorithm for Hyperspectral Images Based on Correlation Coefficients Adaptive 3D Zerotree Coding," IET Image Processing, Vol. 2, 2008, pp. 72-82.

[13] H. Ying and L. Guizhong, "Lossy-to-Lossless Compression of Hyperspectral Image Using the Improved AT-3D SPIHT Algorithm,” 2008 International Conference on Computer Science and Software Engineering, 2008, pp. 963-966.

[14] C. Yushin and W. A. Pearlman, "Quantifying the Coding Performance of Zerotrees of Wavelet Coefficients: Degree-k Zerotree,” IEEE Transactions on Signal Processing, Vol. 55, 2007, pp. 2425-2431. http://dx.doi.org/10.1109/TSP.2007.893218 